\title{
Spreading Awareness of Attenuating Vehicular Speed After Lifting of Covid-19 Lockdown Using Web-Based Estimation of Visual Reaction Time for Primary Prevention of Road Traffic Collision
}

\section{Prashant Rajdeep ( $\sim$ prashant.rajdeep-physiology@msubaroda.ac.in )}

Baroda Medical College, The Maharaja Sayajirao University

\section{Lajja Patel}

Baroda Medical College, The Maharaja Sayajirao University

\section{Steffy CD}

Baroda Medical College, The Maharaja Sayajirao University

Preeti Panchal

Baroda Medical College, The Maharaja Sayajirao University

\section{Research Article}

Keywords: Collision prevention, Reaction time, Cognition, Web, Covid-19 lockdown, Awareness

Posted Date: February 9th, 2022

DOI: https://doi.org/10.21203/rs.3.rs-1238174/v1

License: (c) (i) This work is licensed under a Creative Commons Attribution 4.0 International License.

Read Full License 


\section{Abstract}

Objectives- Attenuating the vehicular speed after lifting of lockdown during Covid-19, by employing visual reaction time (RT) as a tool to prime citizens for creating decorum of driving and checking the road traffic fatalities.

Background- It is indispensable to curb the driving speed post lockdown to avoid accidents. Even though, the impact of inactivity on RT has been well established, an insight into the new method can deal with the gross issue of road traffic casualty worldwide.

Method- Using a web-based platform (http://physicsiology.com), RT was derived by visual stimulation test. Of which, the slowest and the fastest RT were obtained. Using these and prefeed average vehicular speed before lockdown in the website the post lockdown vehicular speed were quantified for the participant.

Apart from spreading awareness the website has also collected data for age and days of lockdown.

Results- Compared to pre lockdown vehicular speed, reduced post lockdown speed was well calculated and suggested. Furthermore, from 643 participants, there was a correlation between RT, age, and days of lockdown.

Conclusion- Containment of speed can be achieved through RT to prime people for prevention of road traffic collision after lifting of Covid-19 lockdown.

\section{Background}

As India leaps towards becoming a developed nation, roadways play a crucial role in development, helping people reach far-off places. India has more than 210 million registered motor vehicles as of 2015, providing ease and comfort in daily commute.(1) But the luxury of having any means of commutation comes with a social responsibility of driving safely and sensibly. Driving is a skilled task that involves a complex interrelation of the brain's motor and cognitive functions and is acquired and stored as working memory with due practice.(2) The key factor in driving is speed; hence, controlling the vehicle speed can mitigate collisions. Every year worldwide, around 1.35 million deaths occur due to road accidents.(1) This is a major part that can logically be attributed to breaking the traffic rules and regulations. After lifting of the Covid-19 lockdown, the vehicles' speed can be unforeseeable, leading to chaos. Lockdown is a rare phenomenon. However, when it occurs, many drivers may lose the accustomed driving practice. Besides, many drivers drive essentially every day on roads without traffic jams, making them get used to driving at high speeds. After lifting of the Covid-19 lockdown, the traffic on roads would be a conflicting image of the one before the lockdown, as people have developed diverse capacities to drive during the lockdown, creating more chaos. An ideal way to prevent such mishaps is by formulating newer means to educate people on regulating vehicle speeds to avoid collisions in such incidences. 
'Visual reaction time' is a simple, non-invasive physiological parameter that gives information about the sensory and motor association.(3) The exact distance at which a vehicle brakes to prevent a collision is dependent on visual reaction time, providing a definite speed to drive at.(4,5) Hence, based on the principle of reaction time, we were able to suggest the effective attenuation of vehicular speed. We propose that more research needs to be carried out to explore the theme's expanse, aiding the administration in facilitating the road mishaps.

\section{Methods}

To measure RT and to check the effect of inactivity on it, which in turn affects the driving, we required a real-time driving test for accurate results. However, this would require sophisticated designed tools or models, which take a lot of expertise and time. Furthermore, due to Covid-19 lockdown it is not feasible. Therefore, we developed an interactive website, '[http://physicsiology.com]', to measure and use RT for quantifying post lockdown vehicular speed for motivating people to reduce their vehicular speed after lifting of lockdown. This will be an easier means to measure RT and also help in maximum participation. We attempted to spread the public awareness by sharing the link on the various social media platforms through self-promotion.

\subsection{Procedure.}

The website's home page had a brief description of the study followed by the tabs; Age, Country, Days of lockdown, and Average speed before lockdown which had to be filled by the participant to undergo test. Subsequently, a visual stimulus test page carrying specific instructions opened up. There, the appearance of a red square or a circle on the screen initiated the test, which was to be clicked with agility until the color turned green on termination of the test. It took twenty-four clicks to complete the test, and the result depended upon the rate at which the participants responded to the stimuli. In the end, the participants had to submit the test, which was followed by a display of their aSRT (Average shortest reaction time), aLRT (Average longest reaction time), and calculated post lockdown vehicular speed. The middle twenty clicks' data were the only ones that were considered for computing post lockdown vehicular speed.

\subsection{Theory/Calculation.}

Quantification of post lockdown vehicular speed can be derived by reaction time obtained by these twenty clicks after sorting them in increasing order. The average shortest reaction time (SRT) were taken from the initial ten reaction times, and the average longest reaction time (LRT) were taken from the last ten responses. The website calculates the average post lockdown vehicular speed using the basic formula for constant speed as given below in Equation (1). 
I.e., Average Speed After Lockdown (ASALD) = [Average Comfortable Speed (ACS) before lockdown X Average Shortest Reaction Time (aSRT)]/Average Largest Reaction Time (aLRT) Equation. (1)

The post lockdown vehicular speed thus obtained was used to counsel the participants to keep their speed in check.

\section{Results}

Apart from spreading awareness, based on the data gathered by the website we had an insight into correlation of RT with age, duration of lockdown.

The distribution of data collected from 643 Indian participants was not normal; hence, a non-parametric test was applied. The test showed that the participants' median age was 32 years, whereas the median days of lockdown were 21 days. The median post lockdown vehicular speed computed using the proposed formula was $7.69 \mathrm{~m} \mathrm{~s}-1(27.7 \mathrm{~km} \mathrm{~h}-1)$, while the average reduction in post lockdown vehicular speed was $4.80 \mathrm{~m} \mathrm{~s}-1$ (17.3 km h-1). Furthermore, response time can be figured out using the table 1.

Table 1. Response time data.

\begin{tabular}{|lll|}
\hline & In seconds & IQR (Inter Quartile Range) \\
\hline Median SRT & 0.45 & 0.41 to 0.52 \\
\hline Median LRT & 0.7 & 0.58 to 1.00 \\
\hline
\end{tabular}

Upon further analysis using the Pearson's coefficient of correlation, the most striking result from the data showed correlation between age and aSRT ( $r=0.24,95 \% \mathrm{Cl} 0.16$ to $0.31, \mathrm{p}<0.0001)$. Similar affirmations can be seen in the study by Woods et al. (6), where we see an increase in latencies with increase in the age. However, the correlation of age with aLRT was non-significant. We see quite a different correlation between the duration of lockdown and aSRT and aLRT, as shown in table 2.

Table 2. Correlation Between Duration of Lockdown and average SRT \& LRT.

\begin{tabular}{|llll|}
\hline Correlation between duration of lockdown and aSRT & $r=-0.11$ & $p=0.007$ & $95 \% \mathrm{Cl}$ (-0.18 to -0.03$)$ \\
\hline Correlation between duration of lockdown and aLRT & $r=-0.04$ & $p=0.27$ & $95 \% \mathrm{Cl}$ (-0.12 to 0.03) \\
\hline
\end{tabular}

\section{Discussion}

Our expertise in navigating traffic makes us judge the vehicular speed and distance between our vehicle and the vehicle ahead of us. Presenting our perspective concerning the variance in visual RT, which is the brain's time to process the information. This involves sequences of events before the action can be implemented, which could be one of the influencers for speed control. Based on the assembly of 
substantial evidence stating that different brain areas work to regulate the speed while driving, $(7,8)$ we derived the tentative post lockdown vehicular speed using the visual RT and pre lockdown speed.

It has been authenticated that the RT increases with increasing age and increase in inactivity duration. (6) The disparity in our results, showing a negative correlation between the RT and duration of inactivity, implies the need for further investigation down the line. The plausible cause of this negative correlation can be due to increased mobile screen time of participants, resulting in exceptional responses, as demonstrated by J. Huang et al.(9) However, the converse can also be true, which is akin to our assumption that inactivity can prolong the RT. Therefore, the negative correlation strengthens our hypothesis that the RT increases with an increase in inactivity duration. Contrary to the expectation, we did not find a significant correlation between aLRT with age $(r=0.04, p=0.34,95 \% \mathrm{Cl}-0.04$ to 0.11$)$ and aLRT with days of lockdown (as in Table 2). The most likely reason for it could be because the shortest RT is a curiosity-driven initial response, while the longest RT is the wearied one. Due to unavailability of pre- lockdown data, we consider SRT as an average RT before lockdown and LRT as an average RT after lockdown. Let us understand our perspective with an example:

Before lockdown, a person $\mathrm{X}$ is riding with a constant ACS of $11.11 \mathrm{~m} \mathrm{~s}-1$ ( $40 \mathrm{~km} \mathrm{~h}-1)$ and at an average $\mathrm{RT}$, which would be equal to SRT $=0.5 \mathrm{~s}$. After lockdown, the same person has an average RT equal to $\mathrm{LRT}=0.7 \mathrm{~s}$. Here, there can be two alternatives -

\section{1-Constant Speed (no acceleration)}

For a rider riding at a constant speed (no acceleration). Distance travelled in time $(\mathrm{SRT})=11.11 \times 0.5=$ $5.555 \mathrm{~m}$; we call it as safety distance. Now for the brain to cruise post lockdown with a reduced RT of 0.7

s. The brain can tackle it in either of the two ways:

To maintain the same safe distance $(5.555 \mathrm{~m})$. The rider should drive at a constant speed of:

$=5.555 / 0.7=7.9357 \mathrm{~m} \mathrm{~s}^{-1}(28.5685 \mathrm{~km} \mathrm{~h}-1)$

Or

To maintain the same constant speed $(11.11 \mathrm{~m} \mathrm{~s}-1)$. The rider should maintain a safe distance of:

$=11.11 \times 0.7=7.777 \mathrm{~m}$

It is unjust to ascribe only the RT for braking while omitting deceleration, as drivers use both back and forth. Hence,

\section{2-The Application of The Brake.}


For rider applying brake (deceleration) and with an average rider, max deceleration $=4.6 \mathrm{~m} \mathrm{~s}-2$.(10). (Even if we consider the negative correlation of RT with the days of lockdown in the present study, the deceleration seen post lockdown would still be higher than the normal; hence contemplating driver maximum would be more appropriate). Distance travelled in time $(\mathrm{SRT})=11.11 \times 0.5-0.5 \times 4.6 \times(0.5) 2=$ $4.98 \mathrm{~m}$ of minimum braking distance or safe distance. Similarly, for the brain to cruise post lockdown with a reduced RT of $0.7 \mathrm{~s}$. The brain can tackle it in either of the two ways:

To maintain the same safe distance $(4.98 \mathrm{~m})$. The rider should attain a speed of:

$=(4.98+0.5 \times 4.6 \times 0.7 \times 0.7) / 0.7=8.724 \mathrm{~m} \mathrm{~s}-1(31.4064 \mathrm{~km} \mathrm{~h}-1)$.

Or

To maintain the same speed (11.11 m s-1). The rider should maintain a distance.

$=11.11 \times 0.7-(0.5 \times 4.6 \times 0.7 \times 0.7)=6.65 \mathrm{~m}$

Nevertheless, every individual has its range of deceleration depending upon their driving skill, type of vehicle and condition of road. This hinders the generalised application of average rider max in countries with poorly regulated traffic.(11) For analogous reasons, it was not implemented in the present study as well. Moreover, it may remain unchanged post lockdown. The calculations given above can be well acknowledged, but with the consideration that acceleration/deceleration cannot be regarded due to lack of feasibility. Maintaining a safe distance at the lowest speed would be the safest approach, coexisting with uniform speed calculation. Thereupon, we proceed with emphasising visual RT and maintaining uniform speed. Therefore, a driver can use either a comfortable speed or safe distance, both conditioned in the brain. Which was kept at a constant range for using simultaneously subconsciously while driving. This speed can be achieved in the best possible way through fine-tuning the RT and acceleration or deceleration. After habituating the traffic movement pattern, the brain can be conditioned to maintain a safe distance and comfortable speed to avoid fatalities. Therefore, lack of practice might affect this automaticity.(2) Hence, we presume that post lockdown in a small group of the population, the RT falls beyond the constant range, with a growing probability of misjudgements and collisions. To forestall this, the driver must make some voluntary adjustments with either the speed or the distance.

\subsubsection{Among the speed or distance, modulation in which of these would yield better safety on the road?}

Recalling our driving lessons, do you remember learning to speed up at the very beginning? Or simply mastering the basics first, which included driving slowly at a safe distance from the other vehicles. Likewise, downscaling the speed would decrease the impact during a collision by abating the momentum. Once we achieve proficiency, we acquire the skill of driving faster and maintaining much less distance from the other vehicles. Therefore, a need for detailed studies should be carried out to 
understand the activity of the brain during and after the driving lessons and how and when it switches from one to another to manipulate speed and distance. We also propose ascertaining a specific centre in the brain that controls the speed. Adhering to our primitive learning behaviour where we give more importance to distance than speed, we would tend to keep a safe distance. Hence, as explained above, maintaining a safe distance at the lowest speed would be the safest approach, coexisting with uniform speed calculations. We have used it to sensitise the public to quantify their speed post lockdown, as patience is something you admire in the driver behind you, but not in the one ahead. Hence, the theme of the study was to heave the quote 'Speed thrills but kills.

\subsection{Shortcomings of our study.}

The difficulty level of the visual stimulus test page was easy, as the number of clicks that provide the response time was limited to just 20 . This gave crude results. In different studies, after excluding scientific errors, the statistically normal RT is $310 \mathrm{~ms}$,(3) while in our study, the technical errors have not been taken into consideration. Moreover, fast drivers and slow drivers can nullify their effects post lockdown and since stringent observation of the traffic rules and regulations in developing countries is difficult, the study's generalised application seems difficult and erroneous.

However, any technique that can reduce the impact or number of collisions will be benevolent to society. As they say, benevolence is absolute, and real kindness costs nothing but means everything.

\section{Conclusion}

We designed the study after speculating the scenario that might occur after lifting of Covid-19 lockdown. Our supposition of utter chaos on the roads due to variation in the reaction time holds by our findings, which correlate the reaction time with the days of lockdown. It could be concluded that there would be interference in the brain due to a lack of driving. There would be slowing down of RT or reflex action or other factors that play a role in driving. This could ultimately lead to collision, as the brain's specific area would not have undergone plasticity to deal with beyond the range of accustomed RT. Therefore, visual RT can be appropriate for consideration as a tool to calculate the attenuated vehicle speed of an individual post lockdown. Hence, by this study, the awareness can be spread to 'Slowdown Post Lockdown' to prevent mishaps.

\section{List Of Abbreviations}

$\mathrm{RT}=$ Reaction time

SRT $=$ The Shortest reaction time

LRT $=$ The Longest reaction time aSRT $=$ Average shortest reaction time 
aLRT = Average longest reaction time

ASALD $=$ Average Speed after Lockdown

ACS $=$ Average Comfortable Speed

$\mathrm{CPU}=$ Central processing unit

$\mathrm{ROCOF}=$ Rate of change of frequency

\section{Declarations}

\section{Ethics approval}

This study was performed in line with the principles of the Declaration of Helsinki. The experimental protocols were approved by the 'Institutional Ethics Committee for Biomedical and Health Research (IECBHR) Medical College \& SSG Hospital, Baroda' of University 'The Maharaja Sayajirao University of Baroda' Vadodara, Gujarat. (No. IECBHR/034-2022).

\section{Consent for participation and publication}

Informed consent was obtained from all subjects and/or their legal guardian(s).

\section{Availability of data and materials}

All the data generated or analysed during this study are included in this published article [and its supplementary information files].

\section{Competing Interests}

The authors declare that they have no competing interests.

\section{Funding}

This research did not receive any specific grant from funding agencies in the public, commercial, or notfor-profit sectors.

\section{Authors' contributions}


PR conceptualized, devised and worked out all the technical details of the project. PR and SC carried out the implementation and performed the analysis under the supervision of PP. The original draft was done by PR. The writing, reviewing and editing was done by PR, LP, after consultation with other authors. The resources were managed by PR \& LP.

\section{Acknowledgements.}

Our profound gratitude to the CEO of 'Kinetic Dreamers,' Mr. Bhavesh Rajdeep, for accelerating our idea to reality by endeavouring in the website's thoughtful design. Chaperoning in the last lap of our expedition by their valuable and constructive suggestions and useful critiques our exceptional prepublication reviewers Dr. Kalpita Shringarpure (M.D. P.S.M, M.S. University), Dr. Vismay Trivedi (PhD. in Applied physics, Fellowship at Bar Ilan University, Israel), Dr. Samir Bhagora (M.D. Physiology, Section Officer; Revenue Dept, Gandhinagar) and Dr. R.K. Patel (M.D. Physiology, M.S. University) have ostensibly reviewed our work deserving our deepest appreciation. We extend our acknowledgement to Amanda D. (Ph.D.) of 'Essaypro.com' for her language editing expertise.

\section{References}

1. W.H.O. Global Status Report on Road Safety 2018: Summary. World Health Organization. 2018.

2. Rostami HR, Ashayeri H. Effects of motor skill practice on reaction time and learning retention in Parkinson's disease. Neurol India. 2009;57(6).

3. Collins LF, Long CJ. Visual reaction time and its relationship to neuropsychological test performance. Arch Clin Neuropsychol. 1996;11(7).

4. Li Y, Chen Y. Driver vision based perception-response time prediction and assistance model on mountain highway curve. Int J Environ Res Public Health. 2017;14(1).

5. Olson PL, Sivak M. Perception-response time to unexpected roadway hazards. Hum Factors. 1986;28(1).

6. Woods DL, Wyma JM, William Yund E, Herron TJ, Reed B. Age-related slowing of response selection and production in a visual choice reaction time task. Front Hum Neurosci. 2015;9(APR).

7. Calhoun VD, Pekar JJ, McGinty VB, Adali T, Watson TD, Pearlson GD. Different activation dynamics in multiple neural systems during simulated driving. Hum Brain Mapp. 2002;16(3).

8. Sakai H, Ando T, Sadato N, Uchiyama Y. Speed-related activation in the mesolimbic dopamine system during the observation of driver-view videos. Sci Rep. 2018;8(1).

9. Huang J, Yan E, Cheung G, Nagappan N, Zimmermann T. Master Maker: Understanding Gaming Skill Through Practice and Habit From Gameplay Behavior. Top Cogn Sci. 2017;9(2).

10. Zhu X, Hu X, Chiu YC. Design of Driving Behavior Pattern Measurements Using Smartphone Global Positioning System Data. Int J Transp Sci Technol. 2013;2(4). 
11. Bokare PS, Maurya AK. Acceleration-Deceleration Behaviour of Various Vehicle Types. In: Transportation Research Procedia. 2017. 